\title{
IJV collapsibility index vs IVC collapsibility index by point of care ultrasound for estimation of CVP: a comparative study with direct estimation of CVP
}

This article was published in the following Dove Medical Press journal: Open Access Emergency Medicine

\section{Haitham Mohammed \\ Jassim' \\ Vamanjore A Naushad ${ }^{2}$ \\ Mohamad Yahya Khatib' \\ Prem Chandra ${ }^{3}$ \\ Mohammed Milad \\ Abuhmaira ${ }^{4}$ \\ Sunil Hassan Koya' \\ Mostafa Sayed Ahmed \\ Ellitthy'}

'Intensive Care Department, AI Wakra Hospital, Hamad Medical Corporation, Doha, Qatar; ${ }^{2}$ Accident \& Emergency Department, Al Khor Hospital, Hamad Medical Corporation, Doha, Qatar; ${ }^{3}$ Medical Research Department, Hamad Medical Corporation, Doha, Qatar; ${ }^{4}$ Department of Internal Medicine, Al Khor Hospital, Hamad Medical Corporation, Doha, Qatar
Correspondence: Vamanjore A Naushad Accident \& Emergency Department, Al Khor Hospital, Hamad Medical Corporation, PO Box 3050, Doha, Qatar Tel +97455902 242

Email nousha87@hotmail.com
Purpose: To compare the bedside ultrasound estimation of internal jugular vein (IJV)-collapsibility index with inferior vena cava (IVC)-collapsibility index and invasively monitored central venous pressure (CVP) in ICU patients.

Design: prospective observational study.

Setting: The study was carried out in the ICU of Al Wakra and Al Khor hospitals of the Hamad Medical Corporation, Qatar. The patients were enrolled from November 2013 to January 2015. Patients: Patients admitted to the ICU with central venous catheter were included.

Material and methods: The A-P diameter, cross-sectional area of the right IJV, and diameter of IVC were measured using bedside USG, and their corresponding collapsibility indices were obtained. The results of the IJV and IVC indices were compared with CVP. The sensitivity, specificity, and positive and negative predictive values were calculated to determine the diagnostic and predictive accuracy of the IJV collapsibility index in predicting the CVP.

Results: Seventy patients were enrolled, out of which 12 were excluded. The mean age was $54.34 \pm 16.61$ years. The mean CVP was $9.88 \mathrm{mmHg}($ range $=1-25)$. The correlations between CVP and IJV-CI (collapsibility index) at $0^{\circ}$ were $\mathrm{r}=-0.484(P=0.0001), \mathrm{r}=-0.416(P=0.001)$ for the cross-sectional area (CSA) and the diameter, respectively, and, at $30^{\circ}$, the most significant correlation discovered was $(r=-0.583, P=0.0001)$ for the CSA-CI and $r=-0.559(P=0.0001)$ for the diameter-CI. In addition, there was a significant and negative correlation between IVC-CI and CVP ( $\mathrm{r}=-0.540, P=0.0001)$.

Conclusion: The IJV collapsibility index, especially at $30^{\circ}$ head end elevation, can be used as a first-line approach for the bedside non-invasive assessment of CVP/fluid status in critical patients. IVC-CI can be used either as an adjunct or in conditions where IJV assessment is not possible, such as in the case of a neck trauma/surgery.

Keywords: internal jugular vein, inferior vena cava, collapsibility index, point of care, ultrasound, central venous pressure, IJV, IVC, CI

\section{Introduction}

Hemodynamic monitoring and rapid assessment of intravascular fluid status for the early, rapid, and effective resuscitation of hypovolemia in critically ill and poly trauma patients is an uphill and a challenging task for the intensivists and emergency physicians. Early goal-directed therapy for reaching an optimal fluid status has been shown to reduce morbidity and mortality in patients with severe sepsis and septic shock. ${ }^{1-5}$ Similarly, early recognition and rapid management of hemorrhage in trauma is essential 
because of the fact that hemorrhage accounts for 30\%-40\% of death in trauma patients. ${ }^{6,7}$

The role of clinical examination in assessing the intravascular volume status of critically ill patients has been found to be unreliable and lacking in accuracy and precision. ${ }^{8-14}$ Studies have shown that vital signs are poor indicators of acute blood loss, shock, and response to treatment in trauma patients. ${ }^{15,16}$ During the early stages of shock, blood pressure or heart rate is not a reliable marker of low volume state. ${ }^{17}$ Similarly, the absence of tachycardia or hypotension does not rule out significant blood loss in the early stages of hemorrhagic shock. ${ }^{18}$ Relying entirely on clinical examination and vital signs are often misleading and may lead to loss of precious time in resuscitation.

Measuring central venous pressure (CVP) by inserting a central venous catheter is the gold standard, but it is invasive, time-consuming, and requires trained personnel. In addition, it is not practical in pre-hospital settings or in an urgent resuscitation scenario, and has its own risks and complications. ${ }^{19-23}$ Various non-invasive methods of measurement of CVP using portable ultrasonography as an alternate approach to invasive CVP measurement have been proposed.

Inferior vena cava (IVC) parameters such as diameter, collapsibility, or caval index, as an indirect measure of CVP to assess the volume status of critically ill patients has been studied extensively, with varying and conflicting results. ${ }^{17,24-29}$ However, in $10 \%-15 \%$ of patients IVC measurement is not possible, due to obesity, abdominal surgical dressings, excessive intra-abdominal gas, large amounts of intra-thoracic air, extrinsic structures compressing IVC, increased intraabdominal pressure, increased pulmonary artery pressure, tricuspid, or pulmonary valve disease..$^{24,30-32}$

Various parameters of the internal jugular vein (IJV) are being increasingly used as an alternative to IVC to indirectly measure the CVP. Examination of the height of jugular venous pulsation has been used as an indirect measure of central venous and right atrial pressure in routine clinical practice, but with a poor sensitivity. Physical examination was accurate only in $50 \%$ of patients in estimating the right atrial pressure..$^{33,34}$

Very few studies have been done on the IJV diameter, cross-sectional area, and their corresponding CI (collapsibility index) by ultrasonography as an indirect measure of CVP. Killu et $\mathrm{a}^{35}$ evaluated the correlation between the IJVCI (diameter) and hypovolemia by POC USG (point of care ultrasonography), they found that a IJV-CI of $\geq 39 \%$ had a sensitivity of $87.5 \%$ and specificity of $100 \%$ in identifying hypovolemia in ICU patients. Akilli et al ${ }^{36}$ measured the IJV-diameter, area, and collapsibility index as an indicator of hemorrhagic shock in healthy blood donors, and found that measurement of IJV and jugular index are reliable indicators of class I hemorrhagic shock. However, they have not correlated their results with invasively measured CVP.

Others have studied various other parameters of IJV, that is the IJV/CCA (common carotid artery) ratio, ${ }^{37,38} \mathrm{AP}$-diameter, ${ }^{39}$ cardiac variation in IJV area, ${ }^{40}$ and ratio of IJV diameter at $30 \% 0^{\circ}$ positions ${ }^{41}$ for the assessment of fluid status by bed side ultrasonography, and showed conflicting results.

We hypothesize that the point of care ultrasound imaging of IJV measurements would correlate with that of IVC measurements and invasively monitored CVP, which in turn predicts the volume status of critically ill patients.

\section{Material and methods}

\section{Study design and settings}

This prospective observational study was carried out in the intensive care units of Al Wakra and Al Khor hospitals of the Hamad Medical Corporation-Doha, Qatar. The patients were enrolled from November 2013 to January 2015.

\section{Study participants}

All patients older than 18 years of age admitted to ICU and who had a CVP catheter that had been already inserted for clinical indications were included. The patients who were on mechanical ventilation, who had a history of radiotherapy or surgery to the neck or chest, pulmonary hypertension, severe tricuspid regurgitation, previous or active upper extremity deep venous thrombosis, and pregnant women were excluded from the study.

\section{Study protocol/measurements} Invasive CVP measurement

CVP is measured using an indwelling central venous catheter (CVC) inserted into IJV or Subclavian vein, and a transducer. CVP is recorded at the mid-axillary line where the transducer is leveled with the phlebostatic axis, this is where the fourth intercostal space and mid-axillary line cross each other, allowing the measurement to be as close to the right atrium as possible.

\section{Investigators}

The study measurements were done by three intensivists who were trained in critical care ultrasound and had an individual 
experience of more than 2 years in bedside ultrasound use. The clinical subjective variability was assessed before the start of the study, and the investigators were blinded for the invasively measured CVP.

\section{Machine}

A sonosite M-Turbo ultrasound machine was used for all the examinations. A linear vascular transuder for IJV imaging (7-13 MHz, $38 \mathrm{~mm}$ ) and a phased array probe for IVC imaging (1-5 MHz, $21 \mathrm{~mm}$ ) was used.

\section{Measurements \\ IJV measurement}

All the measurements were done on Right IJV with the patients initially lying supine at $0^{\circ}$ and later head end elevated at $30^{\circ}$. With the patients in supine position, the USG transducer was placed on the right side of the neck in the transverse plane over RIJV $2 \mathrm{~cm}$ above the sternoclavicular joint. The IJV was identified by the color flow Doppler and compressibility. Care was taken not to compress or obliterate the vein by applying minimal pressure. ${ }^{42}$ When the whole circumference of the vein was visible the measurements were done. The recordings were done for four respiratory cycles. The maximum, minimum AP diameters, and cross-sectional area were estimated and, from this, corresponding CI was derived (Maximum diameter or cross-sectional area (CSA)-minimum diameter or $\mathrm{CSA} /$ maximum diameter or CSA) $\times 100 \%$. All the above measurements were repeated with the head end of patients elevated to $30^{\circ}$ position.

\section{IVC measurements}

With the patients lying in supine position the probe was placed at the subxiphoid area and measurements were taken $2 \mathrm{~cm}$ below the hepatic veins. The maximum and minimum AP diameters were measured over four respiratory cycles, and CI was computed and derived.

\section{Ethical issues}

The study protocol was approved by the local ethics committee of Hamad Medical Corporation. Written informed consent was obtained from all the patients or from a legal guardian whenever appropriate.

\section{Data collection}

The collected data included demographic details, clinical data, and diagnosis at the time of admission. All the data and measurements were recorded in a data collection sheet and then entered into the electronic database for the statistical analysis.

\section{Statistical analyses}

Categorical and continuous variables, IJV and IVC diameters, were presented as frequency (percentage) and mean \pm SD. Descriptive statistics were used to summarize all demographic and other clinical characteristics of the patients. Bedside ultrasound measurements of the IJV, IVC diameters were compared to CVP. The Kolmogorov-Smirnov test or Q-Q Plot, as appropriate, was used to test for normality. The relationship between two quantitative variables was examined using Pearson's or Spearman's rank correlation coefficients. Associations between two or more qualitative variables were assessed using chi-square or Fisher's exact tests, as appropriate. Quantitative data mean values between the two independent groups (CVP $\leq 10$ and CVP $>10)$ were analyzed using unpaired $t$ and Mann-Whitney $U$-tests, as appropriate. Various measures of diagnostic accuracy, such as sensitivity, specificity, positive, and negative predictive values were calculated to determine the diagnostic and predictive accuracy of the IJV collapsibility index in predicting CVP. A receiver operating characteristic (ROC) curve was then computed to derive best suitable cutoff values for the IJV collapsibility index and to assess model discrimination and predictive accuracy. A two-sided $P$-value $<0.05$ was considered to be statistically significant. All Statistical analyses will be done using statistical packages SPSS 22.0 (SPSS Inc., Chicago, IL, USA) and Epi-info (Centers for Disease Control and Prevention, Atlanta, GA, USA) software.

\section{Results}

A total of 70 patients were enrolled, out of which 12 were excluded (severe pulmonary hypertension $=5$, severe tricuspid regurgitation $=4$, mechanical ventilation $=1$, history of neck surgery and radiotherapy $=1$, upper extremities DVT $=1$ ), leaving a final cohort of 58 for analysis. All the patients had a CVP recording and IJV parameter recording. In 12 patients IVC could not be visualized; however, these patients were not excluded from the analyses (Figure 1). The mean age was $54.34 \pm 16.61$ years (range $=20-81$ years), $81 \%$ were male, and septic shock was the most common admission diagnosis. Vasopressor (low-medium dose of nor adrenaline $0.05-0.45 \mu \mathrm{g} / \mathrm{kg} / \mathrm{min}$ ) was required in $29 \%$ of patients . The baseline characteristics and admission diagnosis are summarized in Tables 1 and 2.

The mean CVP was $9.88 \mathrm{mmHg}$ (range $=1-25$ ). The correlations between $\mathrm{CVP}$ and IJV-CI at $0^{\circ}$ were $\mathrm{r}=-0.484$ 


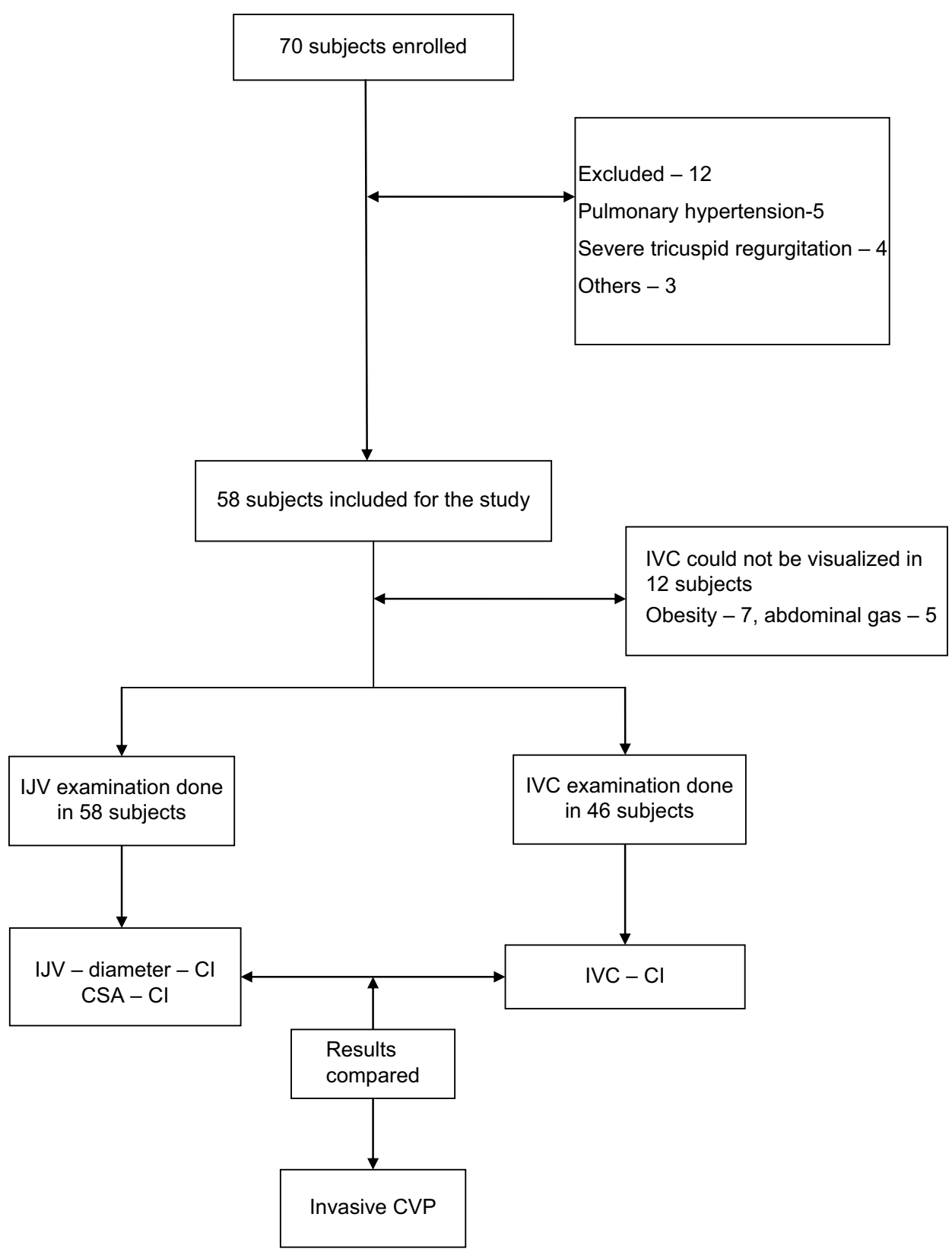

Figure I Flowchart showing subject enrolment

Abbreviations: $\mathrm{Cl}$, collapsibility index; CSA, cross-sectional area; CVP, central venous pressure; IJV, internal jugular vein; IVC, inferior vena cava.

$(P=0.0001)$ for CSA and $\mathrm{r}=-0.416(P=0.001)$ for diameter. However, the highest significant correlation was found between CVP and IJV-CI at $30^{\circ}(\mathrm{r}=-0.583, P=0.0001)$ for CSA and $(\mathrm{r}=-0.559, P=0.0001)$ for diameter. Similarly, IVC$\mathrm{CI}$ also showed a significant correlation with CVP $(\mathrm{r}=-0.540$, $P=0.0001)$. When intergroup correlation was done, it showed a significant correlation between IVC-CI and both the IJV-CI at $30^{\circ}$, whereas IJV-CI at $0^{\circ}$ was poorly correlated (Table 3 ). A linear regression model for the correlation between CVP and collapsibility index are depicted in Figures 2-6.
A CVP of $>10 \mathrm{mmHg}$ was chosen as a clinically significant cutoff value for high CVP level. The test characteristics of ultrasound findings in predicting volume status are given in Tables 1 and 4. The area under the ROC curve (AUC), along with sensitivity, specificity, and PPV, and NPV for IJV collapsibility indices to determine a low CVP $(\leq 10 \mathrm{mmHg})$ are shown in Table 4 and Figure 7 . The most suitable cutoff value determined for collapsibility index for IJV CSA at 0 degree was $>14.1$ with $\mathrm{AUC}=0.792$ with sensitivity, specificity, and PPV ranging $73 \%-82 \%$ in predicting CVP 
$\leq 10$. A cutoff value $>19$ was found to be an optimum for collapsibility index for IJV CSA at $30^{\circ}$ with AUC $=0.851$, with sensitivity, specificity, and PPV ranging $73 \%-83 \%$ in accurately predicting $\mathrm{CVP} \leq 10$.

Table I Comparison of the basic parameters between two groups of CVP

\begin{tabular}{|c|c|c|c|}
\hline Parameters & $\begin{array}{l}\text { CVP } \leq 10 \\
(n=36) \\
(\text { mean } \pm S D)\end{array}$ & $\begin{array}{l}\text { CVP }>10 \\
(n=22) \\
(\text { mean } \pm S D)\end{array}$ & $P$-value \\
\hline Age (years) & $54.47 \pm 17.69$ & $54.14 \pm 15.09$ & 0.941 \\
\hline Weight (kg) & $69.31 \pm 16.65$ & $81.41 \pm 22.87$ & 0.024 \\
\hline Height (cm) & $169.75 \pm 6.90$ & $169.14 \pm 7.29$ & 0.749 \\
\hline Gender (male), n (\%) & 31 (86\%) & $16(72 \%)$ & 0.207 \\
\hline Heart rate & $88.18 \pm 18.25$ & $84.20 \pm 17.55$ & 0.869 \\
\hline MAP & $73.13 \pm 20.21$ & $68.15 \pm 18.17$ & 0.616 \\
\hline $\mathrm{Cl} \mathrm{I}$ index & $26.4 I \pm 16.45$ & $|5.1| \pm|4.6|$ & 0.011 \\
\hline $\mathrm{Cl} 2$ index & $20.00 \pm 16.58$ & $9.92 \pm 7.47$ & 0.010 \\
\hline $\mathrm{Cl} 3$ index & $40.78 \pm 20.75$ & $15.97 \pm 13.75$ & $<0.0001$ \\
\hline $\mathrm{Cl} 4$ index & $26.97 \pm 16.45$ & $10.22 \pm 10.86$ & $<0.0001$ \\
\hline $\mathrm{Cl} 5$ index & $37.10 \pm 19.86$ & $22.59 \pm|5.9|$ & 0.013 \\
\hline
\end{tabular}

Note: $\mathrm{Cl} \mathrm{I}=$ collapsibility index for IJV CSA at $0^{\circ}$ body position; $\mathrm{Cl} 2=$ collapsibility index for IJV diameter at $0^{\circ} ; \mathrm{Cl} 3=$ collapsibility index for IJV CSA at $30^{\circ} ; \mathrm{Cl} 4=$ collapsibility index for IJV diameter at $30^{\circ} ; \mathrm{Cl} 5$, Collapsibility index for IVC at $0^{\circ}$. Abbreviations: CSA, cross-sectional area; CVP, central venous pressure; IJV, internal jugular vein; IVC, inferior vena cava.

Table 2 Showing diagnosis on admission

\begin{tabular}{|l|l|}
\hline Diagnosis & Number \\
\hline Septic shock & 18 \\
\hline Pneumonia & 9 \\
\hline Acute kidney injury & 7 \\
\hline End stage renal disease & 4 \\
\hline COPD exacerbation & 3 \\
\hline Pancreatitis & 3 \\
\hline Myocardial Infarction & 3 \\
\hline Burn & 2 \\
\hline Gastrointestinal hemorrhage & 2 \\
\hline Asthma & 1 \\
\hline Diabetic keto acidosis & 1 \\
\hline Combined medical illnesses and other diagnosis & 15 \\
\hline
\end{tabular}

\section{Discussion}

Our study demonstrated a significant correlation between CI of IJV diameter and CSA at $0^{\circ}$, as well as at $30^{\circ}$ positions with invasively monitored CVP and the measurement at $30^{\circ}$ showing more significant correlation than that at $0^{\circ}$.

Our results validate the results of a previous study by Killu et $\mathrm{al}^{35}$ who investigated the IJV- diameter CI by POC USG as an indicator of hypovolemia in ICU patients. They concluded that IJV diameter CI of $>39 \%$ may be associated with hypovolemia. Their study differed from ours as they measured only IJV diameter CI, and the measurements were done at $30^{\circ}$ positions only.

Few other studies done in the past attempted to demonstrate an association between CVP and various measurements of IJV by ultrasonography. Avcil et al ${ }^{43}$ compared the ultrasound estimation of IJV diameter, area and height, IVC diameter, and index with direct estimation of CVP. The height and diameter of IJV correlated moderately with CVP, whereas IVC diameter and index correlated poorly with CVP. They concluded that the height of RIJV and IVC index has better diagnostic performance for estimating high CVP, whereas IJV area, maximum diameter, IVC maximum diameter showed high sensitivity, and NPV for estimating low CVP levels. ${ }^{43}$ They did not compare the collapsibility index of IJV with CVP. Sean et $\mathrm{al}^{39}$ showed that, if the IJV-AP diameter is between 5.7 and $8.3 \mathrm{~mm}$, then the CVP is $<10 \mathrm{~cm} \mathrm{H}_{2} \mathrm{O}$ and, if it is between 11.2 and $13.8 \mathrm{~mm}$, then the CVP is $>10 \mathrm{~cm}$ $\mathrm{H}_{2} \mathrm{O}$. However, their sample size was less than our study and they included very few patients with higher CVP.

We found that both IJV and IVC parameters by bedside USG can accurately predict the CVP; however, in a few patients the IVC could not be visualized. Very few studies have compared both IJV and IVC measurements together with CVP. Prekker et $\mathrm{al}^{44}$ compared the three modes of point of care USG in predicting CVP, that is maximal IVC diameter, IVC inspiratory collapse, and IJ (Internal Jugular) aspect ratio. They found a good correlation between maximal IVC diameter and IJ aspect ratio however, the one difference between our study and theirs was that they recorded all the

Table 3 Correlation coefficient $(r)$ between CVP and $\mathrm{Cl}$

\begin{tabular}{|l|l|l|l|l|l|l|}
\hline Parameters & CVP & Cl I & Cl 2 & Cl 3 & Cl 4 & CI 5 \\
\hline CVP & I & -0.484 & -0.416 & -0.583 & -0.559 & -0.540 \\
& & $P=0.000 \mathrm{I}$ & $P=0.00 \mathrm{I}$ & $P=0.000 \mathrm{I}$ & $P=0.000 \mathrm{I}$ & $P=0.000 \mathrm{I}$ \\
\hline Cl 5 & & 0.353 & 0.335 & 0.489 & 0.458 & $\mathrm{I}$ \\
& & $P=0.017$ & $P=0.024$ & $P=0.00 \mathrm{I}$ & $P=0.002$ & \\
\hline
\end{tabular}

Note: $\mathrm{Cl} \mathrm{I}=$ collapsibility index for IJV CSA at $0^{\circ}$ body position; $\mathrm{Cl} 2=$ collapsibility index for $\mathrm{JV}$ diameter at $0^{\circ}$; $\mathrm{Cl} 3=$ collapsibility index for $\mathrm{JJV} \mathrm{CSA}$ at $30^{\circ}$; Cl $4=$ collapsibility index for IJV diameter at $30^{\circ} ; \mathrm{Cl} 5=$ collapsibility index for IVC at $0^{\circ}$.

Abbreviations: CSA, cross-sectional area; CVP, central venous pressure; IJV, internal jugular vein; IVC, inferior vena cava. 


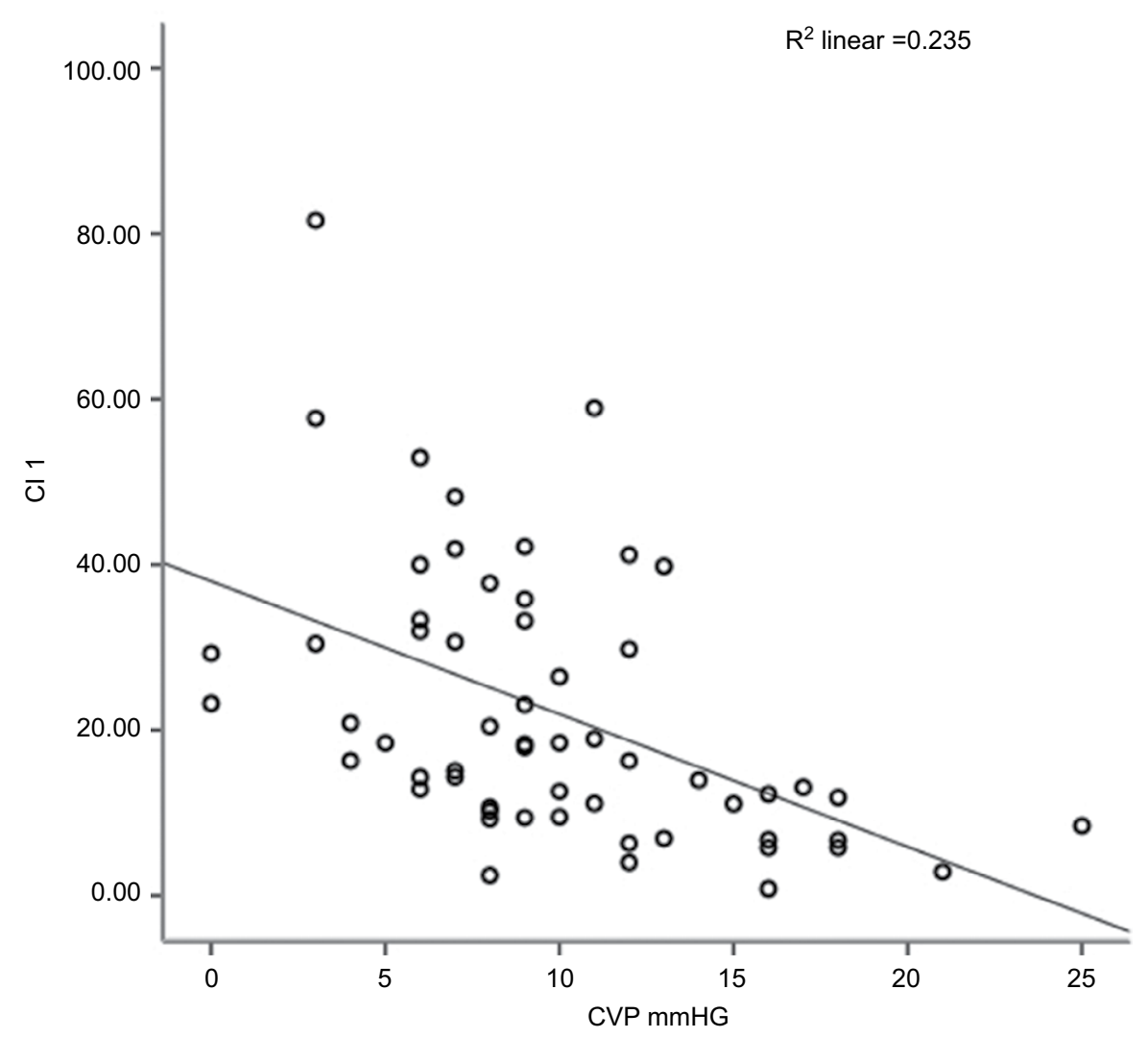

Figure 2 Correlation between CVP and $\mathrm{Cl} I$ (IJV CSA at $0^{\circ}$ ).

Abbreviations: $\mathrm{Cl}$, collapsibility index; CSA, cross-sectional area; CVP, central venous pressure; IJV, internal jugular vein.

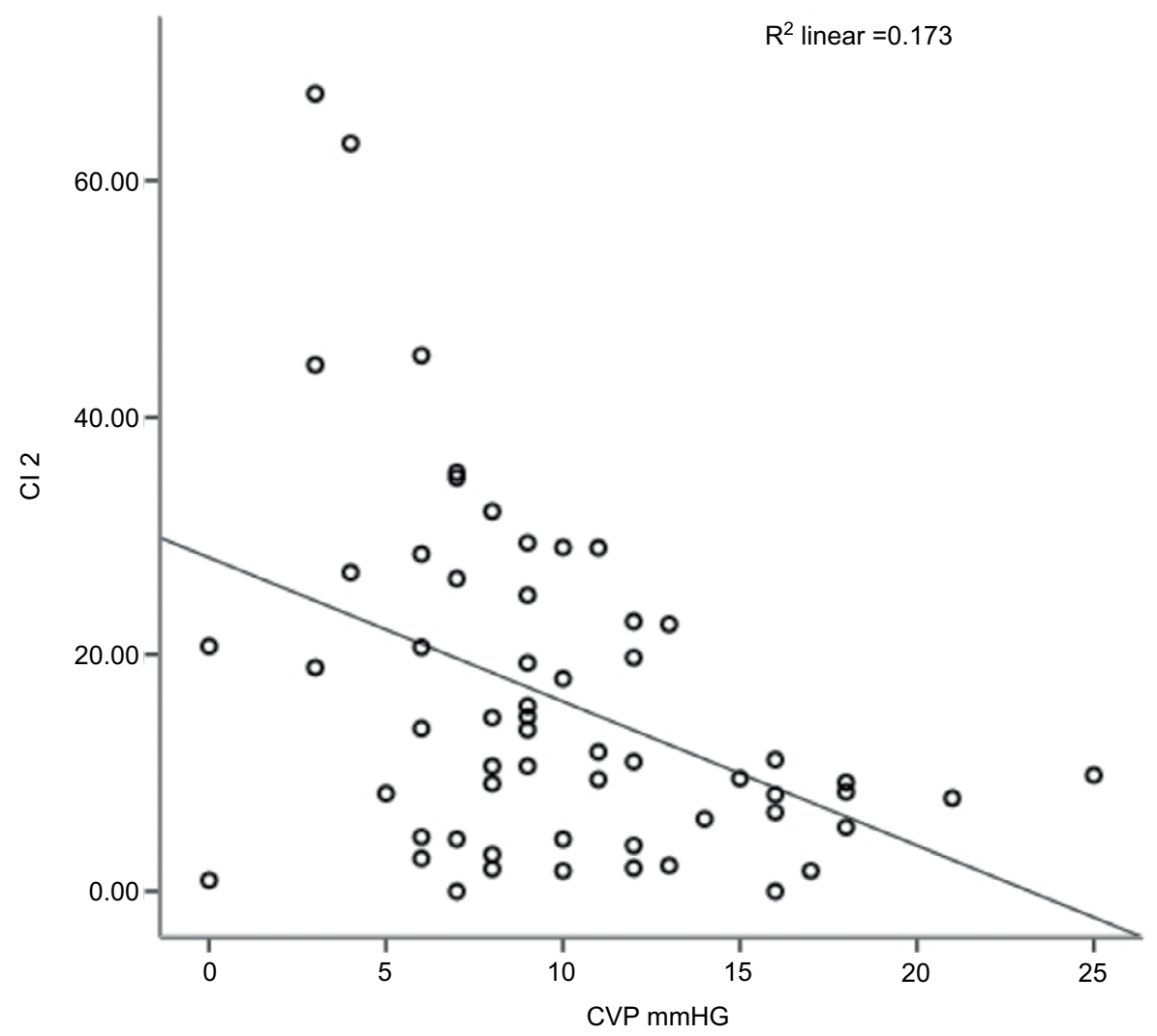

Figure 3 Correlation between CVP and $\mathrm{Cl} 2$ (IJV diameter at $0^{\circ}$ ).

Abbreviations: $\mathrm{Cl}$, collapsibility index; $\mathrm{CVP}$, central venous pressure; IJV, internal jugular vein. 


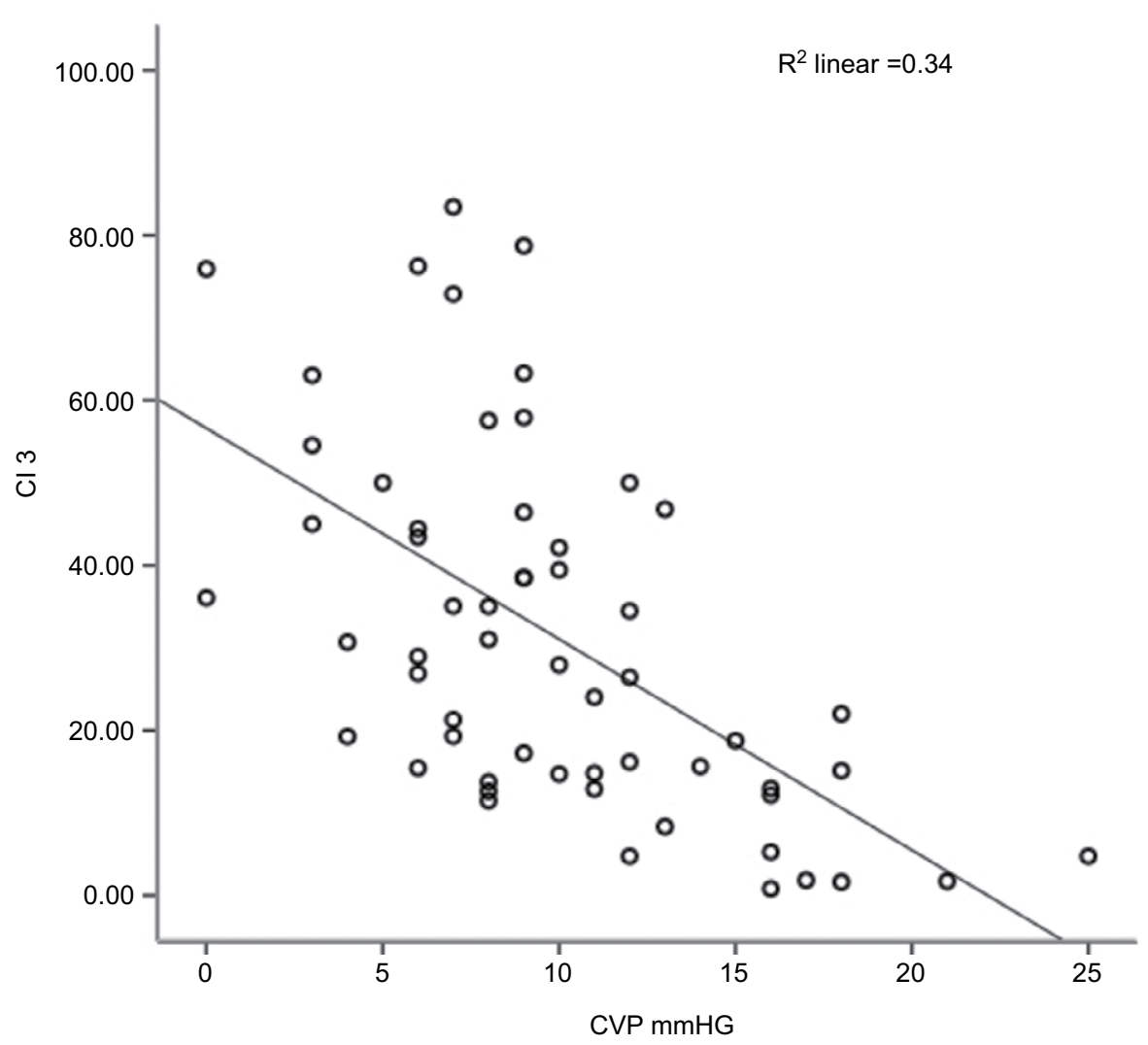

Figure 4 Correlation between CVP and $\mathrm{Cl} 3$ (IJV CSA at $30^{\circ}$ ).

Abbreviations: $\mathrm{Cl}$, collapsibility index; CSA, cross-sectional area; CVP, central venous pressure; IJV, internal jugular vein.

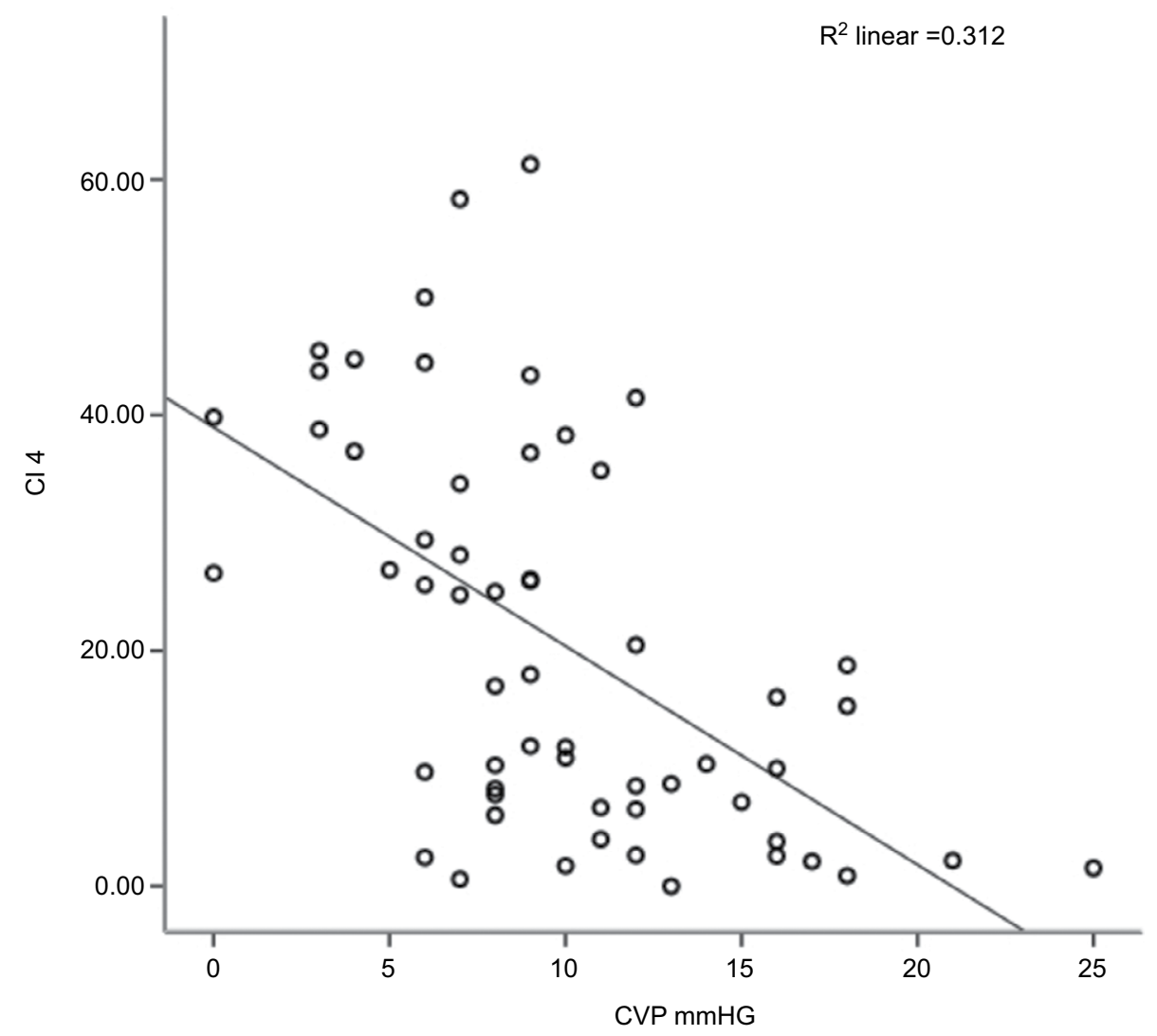

Figure 5 Correlation between $\mathrm{CVP}$ and $\mathrm{Cl} 4$ (IV diameter at $30^{\circ}$ ).

Abbreviations: $\mathrm{Cl}$, collapsibility index; $\mathrm{CVP}$, central venous pressure; JJV, internal jugular vein. 


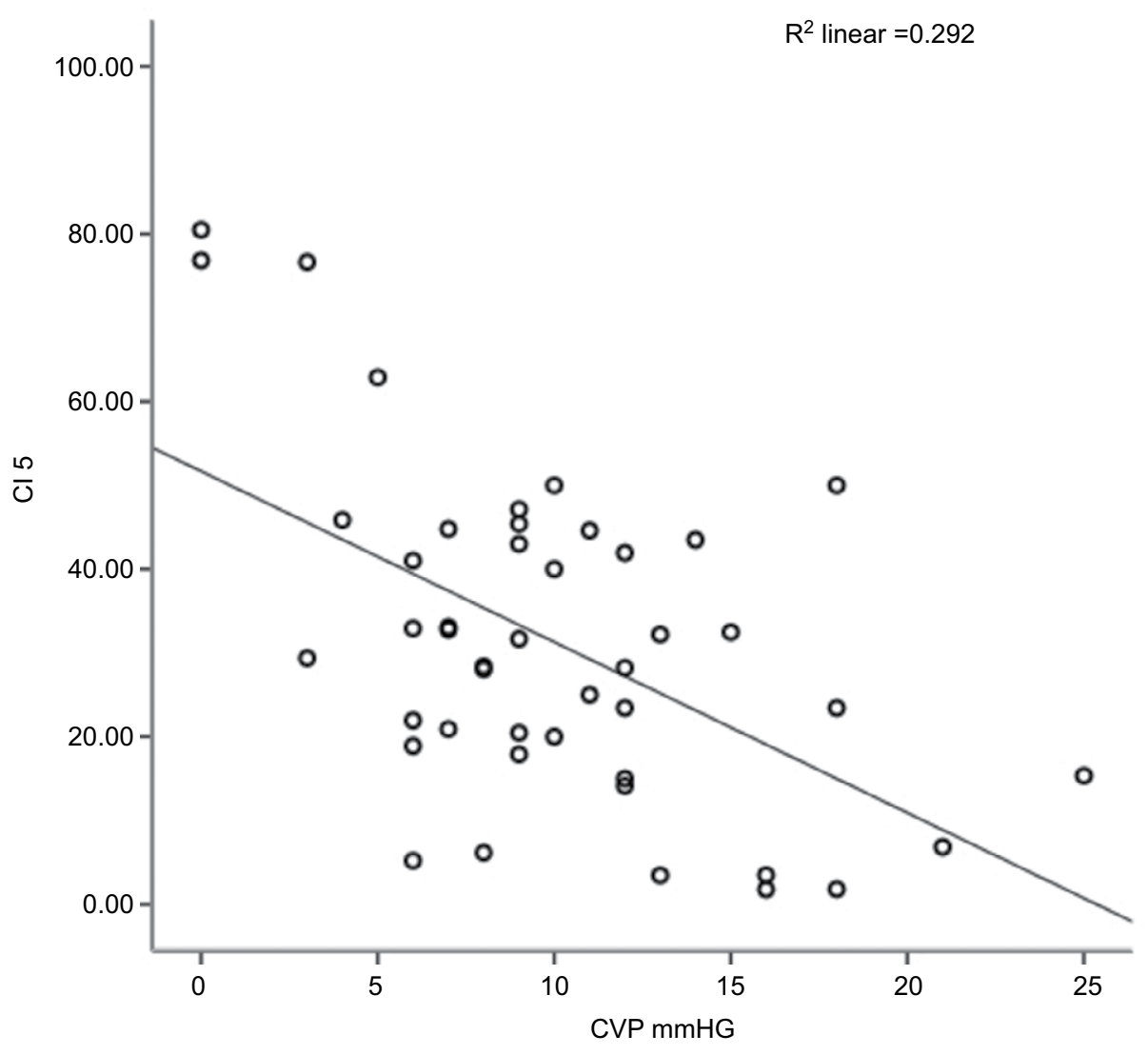

Figure 6 Correlation between CVP and $\mathrm{Cl} 5$ (IVC diameter at $0^{\circ}$ ).

Abbreviations: $\mathrm{Cl}$, collapsibility index; CVP, central venous pressure; IVC, inferior vena cava.

Table 4 Test characteristics of ultrasound findings in predicting CVP $\leq 10 \mathrm{mmHg}$

\begin{tabular}{|l|l|l|l|l|l|l|l|l|}
\hline Parameters & Sensitivity & Specificity & PPV & NPV & Accuracy & AUC & Cutoff & P-value \\
\hline $\mathrm{Cl} \mathrm{I}$ & 77.8 & 72.7 & 82.4 & 66.7 & 75.9 & 0.792 & $>14.1$ & 0.001 \\
\hline $\mathrm{Cl} 2$ & 69.4 & 68.2 & 78.1 & 57.7 & 69.0 & 0.686 & $>10.2$ & 0.018 \\
\hline $\mathrm{Cl} 3$ & 83.3 & 72.7 & 83.3 & 72.7 & 79.3 & $0.85 \mathrm{I}$ & $>19.0$ & $<0.001$ \\
\hline $\mathrm{Cl} 4$ & 80.6 & 68.2 & 80.6 & 68.2 & 75.9 & 0.803 & $>10.2$ & $<0.001$ \\
\hline $\mathrm{Cl} 5$ & 70.4 & 61.1 & 73.1 & 57.9 & 66.7 & 0.711 & $>26.5$ & 0.017 \\
\hline
\end{tabular}

Note: $\mathrm{Cl} \mathrm{I}=$ collapsibility index for $\mathrm{JV} \mathrm{CSA}$ at $0^{\circ}$ body position; $\mathrm{Cl} 2=$ collapsibility index for $\mathrm{JV}$ diameter at $0^{\circ}$; $\mathrm{Cl} 3=\mathrm{collapsibility}$ index for $\mathrm{JV} \mathrm{CSA}$ at $30^{\circ}$; $\mathrm{Cl} 4=$ collapsibility index for IJV diameter at $30^{\circ} ; \mathrm{CI} 5=$ collapsibility index for IVC at $0^{\circ}$.

Abbreviations: AUC, area under ROC curve; CSA, cross-sectional area; CVP, central venous pressure; IJV, internal jugular vein; IVC, inferior vena cava; NPV, negative predictive value; PPV, positive predictive value.

readings in supine positions which might have influenced the results.

Our study showed IJV sonography imaging was easy to obtain in all the patients, whereas IVC imaging was not possible in almost $20 \%$ of patients due to various reasons, of which abdominal gas shadow and obesity were the predominant cause. This finding validates the results of past trials which also showed similar limitations in obtaining the images of IVC. ${ }^{24,30-32}$

Bedside ultrasonography otherwise called point of care ultrasonography has become part and parcel of critical care and emergency departments. Clinicians can rapidly evaluate a critically ill patient with the use of bedside USG without waiting for the advanced imaging. ${ }^{45,46}$ Studies have shown that, with a minimal training of 3 hours, even physicians with less expertise or experience in ultrasonography can accurately take measurements as accurate as physicians having formal trainings. ${ }^{47}$

Assessing the CVP by bedside POC ultrasonography of IJV in emergency and critical settings will have a wide range of practical and clinical implications. First, insertion of a CV 


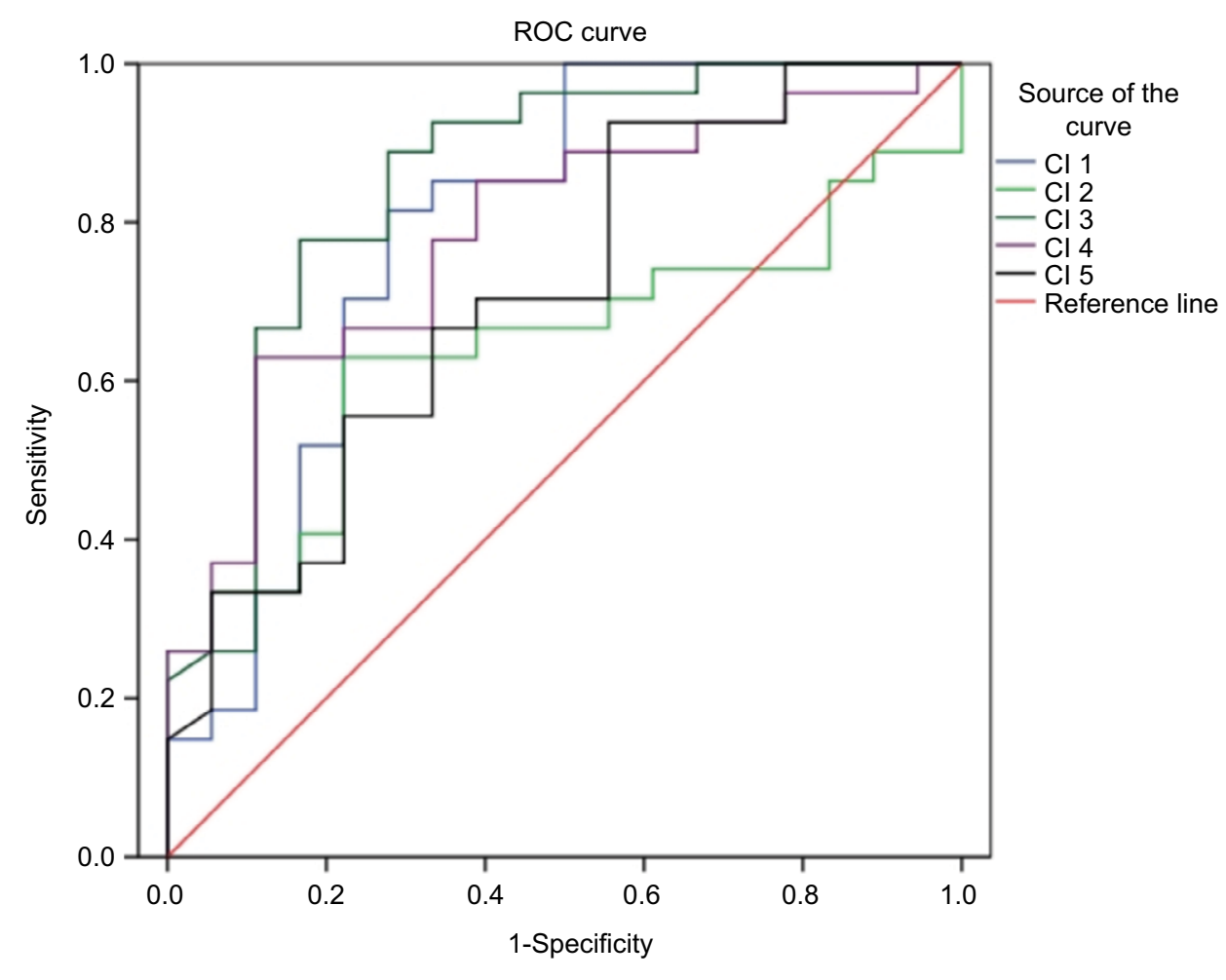

Diagonal segments are produced by ties

Figure 7 ROC curve

Abbreviation: ROC, receiver operating characteristic.

catheter solely for the purpose of measuring CVP in the ICU can be avoided, thereby reducing its potential complications as well as conserving the hospital's resources. Second, in an emergency situation where insertion of a $\mathrm{CV}$ catheter will be time-consuming while resuscitating a critically ill or polytrauma patient, measuring CVP by this method will be an aid to assess the volume status and guide fluid therapy. This is more valuable in centers where the facility for the insertion of a CV catheter is not readily available. Lastly, this method could be used by emergency paramedics in estimating blood loss in trauma patients in out-of-hospital resuscitation with minimal basic training.

\section{Limitations}

The main limitations of our study include the relatively small cohort, and failure to include trauma/surgical patients and mechanically ventilated patients. Our patients included only non-surgical, non-traumatic critically ill patients. Hence, critiques might question whether the results can be applied in polytrauma patients for assessing the CVP. However, we believe that, because of the ease in measuring IJV by bedside ultrasound in such patients, it can be performed if no alternatives are available.

\section{Conclusion}

We demonstrate that POC ultrasonographic measurement of venous parameters of both IJV and IVC provides a useful non-invasive tool for estimating CVP and assessment of intravascular volume status in critically ill patients. Nevertheless, due to the ease of performing, we recommend POC ultrasonographic measurements IJV as a first line non-invasive approach to measure the CVP in the intensive care units as well as in the Emergency departments. IVC measurements can be used in situations where IJV examination is difficult or not practical. We recommend a larger cohort study to substantiate and validate our data.

\section{Disclosure}

The authors report no conflicts of interest in this work.

\section{References}

1. Annane D, Bellissant E, Cavaillon JM. Septic shock. Lancet. 2005;365(9453):63-78. 
2. Dellinger RP, Carlet JM, Masur H, et al. Surviving sepsis campaign guidelines for management of severe sepsis and septic shock. Crit Care Med. 2004;32(3):858-873.

3. Rivers E, Nguyen B, Havstad S, et al. Early goal-directed therapy in the treatment of severe sepsis and septic shock. $N$ Engl J Med. 2001;345(19):1368-1377.

4. Rivers EP, Coba V, Whitmill M. Early goal-directed therapy in severe sepsis and septic shock: a contemporary review of the literature. Curr Opin Anaesthesiol. 2008;21(2):128-140.

5. Jones AE, Focht A, Horton JM, Kline JA. Prospective external validation of the clinical effectiveness of an emergency department-based early goal-directed therapy protocol for severe sepsis and septic shock. Chest. 2007;132(2):425-432.

6. Sauaia A, Moore FA, Moore EE, et al. Epidemiology of trauma deaths: a reassessment. J Trauma. 1995;38(2):185-193.

7. Kauvar DS, Wade CE. The epidemiology and modern management of traumatic hemorrhage: US and international perspectives. Crit Care. 2005;9(suppl 5):S1-S9.

8. Shippy CR, Appel PL, Shoemaker WC. Reliability of clinical monitoring to assess blood volume in critically ill patients. Crit Care Med. 1984;12(2):107-112.

9. Demeria DD, MacDougall A, Spurek M, et al. Comparison of clinical measurement of jugular venous pressure versus measured central venous pressure. Chest. 2004;126(4):747S

10. Marik PE. Assessment of intravascular volume: a comedy of errors. Crit Care Med. 2001;29(8):1635-1636.

11. Eisenberg PR, Jaffe AS, Schuster DP. Clinical evaluation compared to pulmonary artery catheterization in the hemodynamic assessment of critically ill patients. Crit Care Med. 1984;12(7):549-553.

12. Cook DJ. Clinical assessment of central venous pressure in the critically ill. Am J Med Sci. 1990;299(3):175-178.

13. Michard F, Ruscio L, Teboul JL. Clinical prediction of fluid responsiveness in acute circulatory failure related to sepsis. Intensive Care Med. 2001;27(7):1238.

14. Birkhahn RH, Gaeta TJ, Van Deusen SK, Tloczkowski J. The ability of traditional vital signs and shock index to identify ruptured ectopic pregnancy. Am J Obstet Gynecol. 2003;189(5):1293-1296.

15. Gutierrez G, Reines HD, Wulf-Gutierrezme. Clinical review: haemorrhagic shock. CritCare. 2004;8(5):373-381.

16. Martin JT, Alkhoury F, O'Connor JA, Kyriakides TC, Bonadies JA. "Normal" vital signs belie occult hypoperfusion in geriatric trauma patients. Am Surg. 2010;76(1):65-69.

17. Patil P, Kelly N, Papadimos TJ, et al. Correlations between venous collapsibility and common hemodynamic and ventilatory parameters : A multi-variable assessment. OPUS 12. Scientist. 2014;8(1):1-5.

18. Victorino GP, Battistella FD, Wisner DH. Does tachycardia correlate with hypotension after trauma? J Am Coll Surg. 2003;196(5): 679-684.

19. Teichgraber UK, Gebauer B, Benter T, Wagner HJ. Central venous access catheters : radiological management of complications. Cardiovasc Intervent Radiol. 2003;26:321-333.

20. Arenas-Marquez H, Anaya-Prado R, Barrera-Zepeda LM, GonzalezOjeda A. Complications of central venous catheters. Curr Opin Clin Nutr Metab Care. 2001;4(3):207-210.

21. Girbes AR, Polderman KH. Mechanical and infectious complications of central venous catheters. Minerva Anaestesiol. 2003;69:330-332.

22. Wu X, Studer W, Erb T, Skarvan K, Seeberger MD. Competence of the internal jugular vein valve is damaged by cannulation and catheterization of the internal jugular vein. Anesthesiology. 2000;93(2): 319-324.

23. Ruesch S, Walder B, Tramèr MR. Complications of central venous catheters: internal jugular versus subclavian access-a systematic review. Crit Care Med. 2002;30(2):454-460.

24. Nagdev AD, Merchant RC, Tirado-Gonzalez A, Sisson CA, Murphy MC. Emergency department bedside ultrasonographic measurement of the caval index for noninvasive determination of low central venous pressure. Ann Emerg Med. 2010;55(3):290-295.
25. Schefold JC, Storm C, Bercker S, et al. Inferior vena cava diameter correlates with invasive hemodynamic measures in mechanically ventilated intensive care unit patients with sepsis. J Emerg Med. 2010;38(5):632-637.

26. Brennan JM, Ronan A, Goonewardena S, et al. Handcarried ultrasound measurement of the inferior vena cava for assessment of intravascular volume status in the outpatient hemodialysis clinic. Clin J Am Soc Nephrol. 2006;1(4):749-753.

27. Moreno FL, Hagan AD, Holmen JR, et al. Evaluation of size and dynamics of the inferior vena cava as an index of right-sided cardiac function. Am J Cardiol. 1984;53(4):579-585.

28. Arthur ME, Landolfo C, Wade M, Castresana MR. Inferior vena cava diameter (IVCD) measured with transesophageal echocardiography (TEE) can be used to derive the central venous pressure (CVP) in anesthetized mechanically ventilated patients. Echocardiography. 2009;26(2):140-149.

29. Brennan JM, Blair JE, Goonewardena S, et al. A comparison by medicine residents of physical examination versus hand-carried ultrasound for estimation of right atrial pressure. Am J Cardiol. 2007;99(11):1614-1616.

30. Wachsberg RH, Sebastiano LL, Levine CD. Narrowing of the upper abdominal inferior vena cava in patients with elevated intraabdominal pressure. Abdom Imaging. 1998;23(1):99-102.

31. Rein A, Lewis N, Forst L, Gotsman MS, Lewis BS. Echocardiography of the inferior vena cava in healthy subjects and in patients with cardiac disease. Isr J Med Sci. 1982;18:581-585.

32. Brennan JM, Blair JE, Goonewardena S, et al. Reappraisal of the use of inferior vena cava for estimating right atrial pressure. $J$ Am Soc Echocardiogr. 2007;20(7):857-861.

33. McGee SR. Physical examination of venous pressure: a critical review. Am Heart J. 1998;136(1):10-18.

34. Sanders GP, Mendes LA, Colucci WS, Givertz MM. Noninvasive methods for detecting elevated left-sided cardiac filling pressure. $J$ Card Fail. 2000;6(2):157-164.

35. Killu K, Coba V, Huang Y, Andrezejewski T, Dulchavsky S. Internal jugular vein collapsibility index associated with hypovolemia in the intensive care unit patients. Crit Ultrasound J. 2010;2(1):13-17.

36. Akilli NB, Cander B, Dundar ZD, Koylu R. A new parameter for the diagnosis of hemorrhagic shock: jugular index. J Crit Care. 2012;27(5):530.e13-8.

37. Bailey JK, Mc Call J C, Smith S, Kagan RJ. Correlation of internal jugular vein / common carotid artery ratio to central venous pressure: a pilot study in pediatric burn patients. J Burn Care Res. 2012;33:89-92.

38. Hossein-Nejad H, Mohammadinejad P, Ahmadi F. Internal jugular vein/ common carotid artery cross-sectional area ratio and central venous pressure. J Clin Ultrasound. 2016;44(5):312-318.

39. Donahue SP, Wood JP, Patel BM, Quinn JV. Correlation of sonographic measurements of the internal jugular vein with central venous pressure. Am J Emerg Med. 2009;27:851-855.

40. Nakamura K, Qian K, Ando T, et al. Cardiac variation of internal jugular vein for the evaluation of hemodynamics. Ultrasound Med Biol. 2016;42(8):1764-1770.

41. Hilbert T, Ellerkmann RK, Klaschik S, Putensen C, Thudium M. The use of internal jugular vein ultrasonography to anticipate low or high central venous pressure during mechanical ventilation. J Emerg Med. 2016;50(4):581-587.

42. Ciuti G, Righi D, Forzoni L, Fabbri A, Pignone AM. Differences between internal jugular vein and vertebral vein flow examined in real time with the use of multigate ultrasound color Doppler. AJNR Am J Neuroradiol. 2013;34(10):2000-2004.

43. Avcil M, Kapci M, Dagli B, et al. Comparision of ultrasound-based methods of jugular vein and inferior vena cava for estimating central venous pressure. Int J Clin Exp Med. 2015;8(7):10586-10594.

44. Prekker ME, Scott NL, Hart D, Sprenkle MD, Leatherman JW. Pointof-care ultrasound to estimate central venous pressure: a comparison of three techniques. Crit Care Med. 2013;41(3):833-841.

45. Moore CL, Copel JA. Point-of-care ultrasonography. $N$ Engl J Med Overseas Ed. 2011;364(8):749-757. 
46. Ph M, Beaulieu Y, Doelken P, et al. American College of chest Physicians/La Societe de reanimation de Langue Francaise statement on competence in critical care ultrasonography. Chest. 2009;135:1050-1060.
47. Thalhammer C, Siegemund M, Aschwanden M, et al. Non-invasive central venous pressure measurement by compression ultrasound--a step into real life. Resuscitation. 2009;80(10):1130-1136.

\section{Publish your work in this journal}

The Open Access Emergency Medicine is an international, peerreviewed, open access journal publishing original research, reports, editorials, reviews and commentaries on all aspects of emergency medicine. The manuscript management system is completely online and includes a very quick and fair peer-review system, which is all

\section{Dovepress}

easy to use. Visit http://www.dovepress.com/testimonials.php to read real quotes from published authors. 